\title{
$\nabla$ Artificial Intelligence in Medicine and Understanding its Potential for Newer Applications
}

\section{IJCRR}

Section: Healthcare

ISI Impact Factor

(2019-20): 1.628

IC Value (2019): 90.81

$\operatorname{SJIF}(2020)=7.893$

(c) (7) (8)

Copyright@IJCRR

\section{Pathak A ${ }^{1}$, Athavale $\mathrm{H}^{2}$, Pathak $\mathrm{T}^{3}$, Athavale SA}

\author{
'RUHS College of Medical Sciences, ]aipur, India; ${ }^{2}$ School of Computing and Information Technology, Manipal University, Jaipur, India; ${ }^{3} n$ - \\ ternational Institute of Information Technology, Hyderabad, India; ‘Department of Anatomy, All India lnstitute of Medical Sciences, Bhopal, \\ Madhya Pradesh, India.
}

\section{ABSTRACT}

Introduction: Advancements in the performance and efficiency of computers have led to the development of Artificial Intelligence since the advent of the 1950s. The medical field was one of the firsts to seize the opportunity to incorporate these technological advancements into its system. Artificially intelligent technologies were very skilfully added into everyday medical practice enhancing diagnostics and radiological capabilities, bolstering pharmaceutical processes, and revamping several other spheres of medicine. During the chaotic period of the pandemic too, it has proved to be an essential tool.

Aim: For identifying sources of literature, initially google scholar database was used using broad research terms that were aligned to the topic and research question. More focussed terms were identified and were utilised with the 'and' command to perform a database search in Pubmed and Science direct. Cross-references were identified from the articles.

Articles were scrutinized for content and summarized to discuss the utility of this technology in specific areas of medicine and its potential for the future.

Conclusion: Artificial Intelligence has already been forayed into the field of medicine. It has exhibited immense potential in interpreting large amounts of data, deriving breathtaking algorithms, offering pragmatic and cost-effective approaches for prevention, diagnosis and treatment in almost all fields of medicine.

Key Words: Chatbots, COVID19, Deep learning, Machine learning, Neural networks

\section{INTRODUCTION}

Artificial Intelligence, commonly known as machine intelligence is the ability of a machine to analyse the task assigned which subsequently enhances the ability of the machine to successfully achieve its goals. ${ }^{1}$ Artificial intelligence can also be regarded as an oxymoronic term (when coupled with machine learning) which suggests the ability of machines to demonstrate qualities of higher living beings such as flawless cognitive brain function, learning and problem solving which is most often associated with the human brain. ${ }^{2}$ Modern machinery has embodied artificial intelligence to the extent of them being able to understand human speech, autonomously operate motor vehicles, prove to be a valuable screening tool for disease detection, etc. ${ }^{3,4}$ This is possible due to recent progress in extensive digital data acquisition and the wonders of machine learning. ${ }^{4}$ Machine learning, a subdivision of artificial intelligence deals with the under- standing and inference of patterns in a data set. This helps to derive algorithms that are specific to the training data. ${ }^{5}$ Deep learning, a subset of machine learning can understand multiple hidden layers of the training data and thus helps to generate very high accuracy predictive outputs. ${ }^{6}$

Machine learning is divided into three types: Unsupervised, Supervised, and reinforcement. Supervised learning generates algorithms using a known dataset (which is labelled beforehand) which is then used to predict the desired outcome. Unsupervised learning comprises of unearthing hidden patterns from unknown data sets thereby aiding in identifying novel disease mechanisms, genotypes, or phenotypes; the objective ultimately being able to find appropriate solutions without human intervention. Reinforcement learning can be seen as a hybrid between supervised and unsupervised machine learning.?

Since the advent of artificial intelligence in the mid-1950s, much progress has been made. ${ }^{8}$ The application of artificial

\section{Corresponding Author:}

Sunita Athavale, Department of AnatomyAll India Institute of Medical Sciences Bhopal, Madhya Pradesh, India.

Phone: 8878895453; Email: sunita.anatomy@aiimsbhopal.edu.in

ISSN: 2231-2196 (Print)

ISSN: 0975-5241 (Online)

Received: 10.01 .2021

Revised: 26.02 .2021

Accepted: 11.03 .2021

Published: 30.08 .2021 
intelligence and machine learning is highly sought after in the field of medicine. The primary goal concerning healthrelated AI applications is to analyse the working relationship between prevention, screening, and treatment techniques which is then tallied with patient prognosis and clinical outcome. ${ }^{9}$ Currently, immense potentials are being explored in the diagnostic processes, enhanced treatment protocols, drug development in pharmaceutical firms, patient monitoring, and care. ${ }^{10}$

For identifying sources of literature, initially google scholar database was used using broad research terms that were aligned to the topic and research question. The terms used were: Artificial Intelligence, AI in Medicine, History of AI, Machine learning, Deep learning, Neural Networks, AI in Diagnostics, AI in Cardiology, AI in radiology and imaging, $\mathrm{AI}$ in genetics, Chatbots, Big data public health, $\mathrm{AI}$ in Radiology, AI in pharmacology, COVID-19, AI in COVID-19, Drug Design, AI in oncology, Newer applications of AI. More focussed terms were identified from the articles searched in google scholar and were utilised with the 'and' command to perform a database search in Pubmed and science direct. Cross-references were identified from the articles. These articles were analysed using the following criteria for inclusion.

1. The source article should be aligned with the purpose of review

2. The article should be published in a peer-reviewed journal.

3. Effort was made to include recently published articles.

Articles were scrutinized for content and summarized to discuss the utility of this technology in specific areas of medicine and its potential for the future.

\section{ARTIFICIAL INTELLIGENCE INTERWOVEN WITH DIAGNOSTIC MEDICINE}

Diagnostic medicine is a field that encompasses medical techniques designed to detect infections, conditions, and diseases. The institute of medicine at the National Academics of Science, Engineering, and Medicine reports that diagnostic errors contribute to approximately $10 \%$ of patient deaths, and also account for $6-17 \%$ of hospital complications. ${ }^{11}$ These mishaps and shortfalls prompted the integration of artificial intelligence into the world of medicine. The first use of artificial intelligence was in the form of chatbots. Chatbots analyse the symptoms put forward by the user which is then cross-referenced against a database of diseases. In response, the machine will recommend a course of action which is most suitable to the patient's history and patient circumstances. In addition to the prior chatbot technologies, these techniques have now been upgraded to monitor and record vitals such as heart rate and cholesterol level. ${ }^{12} \mathrm{AI}$ is being used in the field of oncology where early detection is key to the prognosis of the patient. ${ }^{13}$ It has achieved commendable accuracy for breast cancer screening. ${ }^{14}$ Deep neural networks - which are a recognized subset technology of machine learning - have been able to scan for and successfully locate enlarged lymph nodes or colonic polyps in computed tomography (CT) images. ${ }^{15,16} \mathrm{~A}$ breakthrough has also been achieved in the medical application of whole slide imaging which has resulted in the formulations of histopathological diagnose ${ }^{17}$ Deep learning is also in the process of being able to decipher the molecular status of a tumour such as assessment of tumour marker proteins, namely, HER2 from pathological data. ${ }^{18}$ Moreover, artificial intelligence is being used in cancer genomics wherein a supercomputer can analyse and identify up to 100,000 genomic mutations and provide precision care for each tumour sample. ${ }^{19}$ This development has revolutionised cancer treatment due to computers capable of employing gold standard treatment options specifically based on the expression of molecular markers and tumour cell mutations and characteristics. ${ }^{20}$ Extending from the field of oncology, artificial intelligence has established a strong foothold in the field of cardiology as well. ${ }^{21}$ These techniques play a critical role in improving the precision of cardiovascular medicine by forming functional phenotypes like electrocardiography, echocardiograms, demographics, haemodynamics, and imaging data. ${ }^{22,23,24}$ Also, molecular profiles from large collections of data and medical records of patients comprising of laboratory test results, physician notes and other relevant information of disease, treatment, and epidemiology may be mined for analyzing association and building predictive models on prognosis and learning drug responses. A recent example of an exemplary use of machine learning in cardiology has been demonstrated by Shah which predicts the prognosis of patients with heart failure and preserved ejection fraction (EF). This is an example of unsupervised machine learning which used 46 different data points to identify intrinsic structures among patients with this particular type of heart failure.$^{25}$ Furthermore, AI has been used in cardiac imaging with great success too. 3D echo data sets acquisition has been fed to computational systems which automatically have been able to identify the heart's anatomy and suitably modify it further for optimal standard views of presentation. ${ }^{22}$ Not surprisingly, the use of artificial intelligence has been interwoven with mainstream medical practice. A machine learns and applies diagnostic tools by understanding patterns and formulating algorithms similar to how a doctor approaches diagnostic challenges. ${ }^{26}$ $\mathrm{AI}$ and machine learning have proved to be successful in analysing and diagnosing skin lesions (including melanoma) as precisely as expert dermatologists. ${ }^{27}$ This software could be added to smartphones whose reach is significantly farther than expert dermatologists. Other fields of medicine that are worth mentioning under the umbrella of fields using machine learning are pulmonary medicine, rheumatology, ophthalmology, otorhinolaryngology, head and neck surgery, etc. 


\section{ARTIFICIAL INTELLIGENCE INTERWOVEN WITH RADIOLOGY AND RADIOTHERAPY}

Another major field of medicine that has seen significant advances is the use of artificial intelligence in the field of radiology. Radiology, as a branch, deals with the detection, characterisation, and subsequent monitoring of disease. Detection in radiology involves manual expertise to identify abnormalities and cognitive skills to reach a diagnosis. Characterisation involves the process of segmentation, diagnosis and staging of the disease. Lastly, monitoring encompasses the evaluation of the treatment response..$^{28}$ Radiology is a branch that is heavily dependent on machines. The first use of computer programs and artificial intelligence was in the form of magnetic resonance imaging (MRI) and positron emission tomography (PET) scans which facilitated improvement in the diagnostic capabilities of physicians and hence their treatment modalities. ${ }^{29}$ But identification and analysis of such scans required expert intervention. Due to the lack of sufficient trained radiologists, enhanced AI systems have been seamlessly integrated within the branch which would increase efficacy, minimize errors and achieve targets with nominal manual input which would provide radiologists with labelled and identified images for faster diagnosis. ${ }^{30}$ Furthermore, deep learning algorithms can learn feature representations from data without human intervention. Deep learning can thus quantify phenotypic characteristics of human tissues, improving diagnosis and clinical care. For example, deep learning can extract predefined features and accurate segmentation of diseased tissues which falls under the pretext of detection. ${ }^{31}$ Further, via the use of carefully laid algorithms and deep learning, the machine can characterise the lesion which helps in the formulation of a diagnosis and its staging, if it involves neoplastic growth and/or cancers. ${ }^{32}$ In the long run, deep learning also aids in the monitoring of the patient which reduces significant work pressure over the healthcare professional and expert radiologists.

\section{ARTIFICIAL INTELLIGENCE INTERWOVEN WITH PHARMACEUTICALS AND DRUG TRIAL RESEARCHES}

From the ideation of using artificial intelligence for improving prescribing techniques to the evolution of personalised medicine, the pharmaceutical industry has integrated itself with artificial intelligence. ${ }^{33,34}$ In particular, the pharmaceutical industry has been known to use $\mathrm{AI}$ in improving its drug manufacturing processes. ${ }^{35} \mathrm{AI}$ has been used to shorten design time, reduce wastage of raw materials, and much more. Human intervention has also been significantly reduced since the use of AI. Concurrently, AI has also aided the industry in drug discovery and design formulations by interpreting and integrating large amounts of patient data and comparing it with randomised controlled trials to judge its efficacy. ${ }^{36} \mathrm{In}$ terestingly, AI has also aided in biomedical and clinical data processing which helps them to assess the efficacy of products launched by pharmaceutical companies. More recently, pharmaceutical companies have devised software that aid in tackling rare diseases and developing personalised medicines based on individual patient's test results, reactions to past medications, and their progress of the disease. ${ }^{37,38}$ This data collected is used to predict treatment results that have huge time \& cost-saving applications. This shows that the addition of artificial intelligence has been a boon to the industry.

\section{ARTIFICIAL INTELLIGENCE INTERWOVEN WITH SMART ELECTRONIC HEALTH RE- CORDS}

Electronic health records (by themselves) are large collections of medical data like patient demographics, medical images, medical notes and prescriptions which are often viewed by people as huge, monolithic and tedious to use. Due to the labour-intensive nature of the use of EHR, they have limited generalisability across databases. ${ }^{39}$ When integrated with artificial intelligence and machine learning, electronic health records can be very easily accessible. ${ }^{40}$ It has changed the data analytic modelling framework from human-driven to data-driven construction. The core reason for the integration of AI and machine learning into the field of EHR is the presence of large and complex datasets in healthcare that require stringent monitoring which is most efficiently managed by $\mathrm{AI}$ algorithms. ${ }^{39} \mathrm{It}$ is a fact that the $\mathrm{AI}$ applications in electronic health records are narrow and premature, but currently, they include data extraction from free text, diagnostic and predictive algorithms facilitating the development of predictive models which warn physicians of high-risk conditions such as heart failure and sepsis, facilitate clinical documentation and data entry, strengthen clinical decision support consisting of computer algorithms which recommend treatment strategies, etc..$^{39,41,42}$

\section{ARTIFICIAL INTELLIGENCE INTERWOVEN WITH PUBLIC HEALTH:}

Apart from the use of $\mathrm{AI}$ in diagnostics, screening and risk prediction, it has also transformed the conventional public health care systems to make superior healthcare accessible to all members of the community. One of the most important uses of AI in the public health setting is increasing patient adherence and access to treatment. ${ }^{9}$ AI algorithms are used to identify patterns in population clusters, especially comprising of women, infants and people below the poverty line helping them to make sure that they diligently follow their treatment regimes which have massive implications in the 
control and eradication of a particular disease. ${ }^{43} \mathrm{AI}$ has also boosted patient adherence by providing valuable data by running predictive analysis algorithms which aid in improving the outreach of nationally run treatment programmers. Lately, AI has also been used to track the progress of grass root level health care workers who operate in geographies with dense and unmonitored populations. AI aids in actively forming algorithms that streamline data collection in the field and analyse on ground conditions on whether appropriate healthcare efforts are being channelized to the welfare of the community. Interestingly, it has also been used in the prediction and containment of epidemics. By using machine learning, particularly the unsupervised variant, algorithms are formulated to monitor information from the news, official health care reports and even the social media in several languages around the world, red-flagging where high priority diseases are mentioned. ${ }^{44}$ These trends are then monitored by algorithms which then alerts the authorities once it identifies a significant threat level in the community.

\section{ARTIFICIAL INTELLIGENCE INTERWOVEN WITH DIAGNOSTICS AND THERAPEUTICS OF COVID-19}

The pandemic of 2020 wherein the causative agent was the SARS-nCoV19 virus caused havoc and lead to the disruption of the world order. ${ }^{45}$ This lead to the search for novel diagnostic and therapeutic methods which warranted the use of artificial intelligence. ${ }^{46,47}$ It is known that artificial intelligence and machine learning (specifically models trained using unsupervised learning) with their strong pattern detection capabilities are great tools for the early detection of viruses and diagnosis of diseases. Recent applications focus on predicting mutations before the occurrence of a new strain by applying rough set theory as a processing tool for imprecise information. ${ }^{48}$ In addition to the prediction of mutations, AI can help with cost and time effective methods for detection of covid-19 by using algorithmic structures like random forests, decision trees and support vector machines. ${ }^{49}$ Moreover, AI aids in developing vaccines and treatments at a significantly faster rate compared to regular clinical trials under powerful optimisation techniques of contemporary machine learning models. ${ }^{50}$ With internet and GPS enabled mobile devices being even more accessible to large populations, contact tracing has been made easier than ever with the help of ML-fueled data visualisation and analytics. ${ }^{51}$

\section{NEWER APPLICATIONS OF ARTIFICIAL INTELLI- GENCE AND MACHINE LEARNING IN MEDICINE}

To date, newer strides are being made in the field of artificial intelligence and its application in the field of medi- cine. Some prodigious advancement has been made wherein a plausible concept has been put forward where the health status, comprising of the estimation of hormone levels and physiological state of a person is assessed by deciphering speech samples including but not limited to the vocal consonants (VC), consonant-vowel (CV), environment formants of the utterance, speech quality of the utterance, pitch of the utterance etc. ${ }^{52,53}$ The system consists of a processor and a memory paired with the processor. The processor executes programmed instruction for isolating one or more phonation segments which are analysed and cross-matched by the processing unit to provide corresponding hormone levels of the person based on different speech features. ${ }^{52,53}$ Significant strides have been made in the field of robotic surgery too, where raven robots and PR2 robots are being used in the future automation, deep learning for the comprehensive evaluation of surgical skills and techniques, and machine learning for enhancing surgical robotic materials which improve the dexterity of surgeries requiring immense precision. ${ }^{54}$ The use of $\mathrm{AI}$ in the field of surgery has significantly improved pre and post-operative experience for the patient. ${ }^{55}$ Owing to the diverse applicability of AI systems, automated diagnoses or computer-aided diagnosis (CAD) are being devised which train using huge amounts of patient data, physiological signals and images based on meticulous use and analysis whose application is being noted in the field of neurology, neurosurgery, radiology, and others. ${ }^{56}$ Lastly, the newest breakthrough has been noted in the sub-set of artificial intelligence termed computer vision. ${ }^{57}$ Computer Vision in the field of artificial intelligence that trains the computer to interpret and understand the visual world. Data from images are extracted by various information retrieval algorithms by analysing information from individual pixels and transforming them into computer-readable and manipulatable data. This transformed data can then be used as any regular data set to train/test machine learning models and deploy them for real-world applications. ${ }^{58}$ Some applications including but not limited to can be, monitoring heart rate by using high definition video samples from the neck region detecting \& classifying bone fractures, tumours, and soft tissue conditions by leveraging machine learning models generated by huge amounts of historical data are being noted. ${ }^{59,60,61}$

\section{CONCLUSION}

The evolution of the use of artificial intelligence and machine learning technology has revolutionised how the world of medicine is perceived and understood. It has brought forward breathtaking algorithms that are capable of interpreting large amounts of data and provide the most pragmatic and useful treatment or production options which ultimately benefits mankind which is the ultimate goal of the pursuit of the practice of medicine. Hence, AI technology should be 
welcomed in the field of medicine with open arms.

\section{ACKNOWLEDGEMENT}

The authors acknowledge the immense help received from the scholars whose articles are cited and included in references of this manuscript. The authors are also grateful to authors, editors and publishers of all those articles, journals and books from where the literature for this article has been reviewed and discussed.

Conflict of Interest - None

Source of Funding - None

\section{AUTHORS CONTRIBUTION:}

Pathak A - Conceptualisation, Literature search, Manuscript writing

Athavale $\mathrm{H}$ - Literature search and review, Manuscript writing

Pathak T - Literature search and review, Manuscript writing

Athavale SA - Conceptualisation, Review, Final editing of the manuscript

\section{REFERENCES}

1. Hamet P, Tremblay J. Artificial intelligence in medicine. Metab. 2017;69S: S36-S40

2. Russell S. Rationality and intelligence. Artificial Intellig. 1997;94(1-2):57-77.

3. McKinney S, Sieniek M, Godbole V, Godwin J, Antropova N, Ashrafian $\mathrm{H}$ et al. International evaluation of an AI system for breast cancer screening. Nature. 2020;577(7788):89-94.

4. $\mathrm{Yu} \mathrm{K}$, Beam A, Kohane I. Artificial intelligence in healthcare. Nat Biomed Eng. 2018;2(10):719-731.

5. Deo R. Machine Learning in Medicine. Circul. 2015;132(20):1920-1930.

6. McBee M, Awan O, Colucci A, Ghobadi C, Kadom N, Kansagra A et al. Deep Learning in Radiology. Acad Radiol. 2018;25(11):1472-1480.

7. Libbrecht M, Noble W. Machine learning applications in genetics and genomics. Nat Rev Genet. 2015;16(6):321-332.

8. Kaul V, Enslin S, Gross S. History of artificial intelligence in medicine. Gastrointest Endosc.. 2020;92(4):807-812.

9. Davenport $\mathrm{T}$, Kalakota $\mathrm{R}$. The potential for artificial intelligence in healthcare. Future Healthc J. 2019;6(2):94-98.

10. Shaban-Nejad A, Michalowski M, Buckeridge D. Health intelligence: how artificial intelligence transforms population and personalized health. Nat Phy J Digit Med. 2018;1(1):45-49.

11. Committee on Diagnostic Error in Health Care; Board on Health Care Services; Institute of Medicine; The National Academies of Sciences, Engineering, and Medicine; Balogh E, Miller B, Ball J, editors. Improving Diagnosis in Health Care. Washington (DC): National Academies Press (US); 2015 Dec 29.

12. Mierzwa S, Souidi S, Conroy T, Abusyed M, Watarai H, Allen T. On the Potential, Feasibility, and Effectiveness of ChatBots in Public Health Research Going Forward. Online J Public Health Inform. 2019;11(2): 321.
13. Kann BH, Thompson R, Thomas CR Jr, Dicker A, Aneja S. Artificial Intelligence in Oncology: Current Applications and Future Directions. Oncology (Williston Park). 2019;33(2):46-53.

14. Houssami N, Kirkpatrick-Jones G, Noguchi N, Lee C. Artificial Intelligence (AI) for the early detection of breast cancer: a scoping review to assess AI's potential in breast screening practice. Expert Rev Med Dev. 2019;16(5):351-362.

15. Urban G, Tripathi P, Alkayali T, et al. Deep Learning Localizes and Identifies Polyps in Real Time With 96\% Accuracy in Screening Colonoscopy. Gastroent. 2018;155(4):1069-1078.

16. Ehteshami Bejnordi B, Veta M, Johannes van Diest P, van Ginneken B, Karssemeijer N, Litjens G et al. Diagnostic Assessment of Deep Learning Algorithms for Detection of Lymph Node Metastases in Women With Breast Cancer. J Ame Med Ass. 2017;318(22):2199.

17. Campanella G, Hanna M, Geneslaw L, Miraflor A, Werneck Krauss Silva V, Busam K et al. Clinical-grade computational pathology using weakly supervised deep learning on whole slide images.

18. Nat Med. 2019;25(8):1301-1309.

19. Vandenberghe M, Scott M, Scorer P, Söderberg M, Balcerzak D, Barker C. Relevance of deep learning to facilitate the diagnosis of HER2 status in breast cancer. Sci Rep. 2017;7(1).

20. Xu J, Yang P, Xue S, Sharma B, Sanchez-Martin M, Wang F et al. Translating cancer genomics into precision medicine with artificial intelligence: applications, challenges and future perspectives. Hum Genet. 2019;138(2):109-124.

21. Shimizu H, Nakayama K. Artificial intelligence in oncology. Cancer Sci. 2020;111(5):1452-1460.

22. Johnson K, Torres Soto J, Glicksberg B, Shameer K, Miotto R, Ali $\mathrm{M}$ et al. Artificial Intelligence in Cardiology. J Am Coll Cardiol. 2018;71(23):2668-2679.

23. Zhang J, Gajjala S, Agrawal P, Tison G, Hallock L, BeussinkNelson L et al. Fully Automated Echocardiogram Interpretation in Clinical Practice. Circulation. 2018;138(16):1623-1635.

24. Kao D. Intelligent Artificial Intelligence: Present Considerations and Future Implications of Machine Learning Applied to Electrocardiogram Interpretation. Circ Cardiovasc Qual Outcomes. 2019;12(9):e006021.

25. Siegersma K, Leiner T, Chew D, Appelman Y, Hofstra L, Verjans J. Artificial intelligence in cardiovascular imaging: state of the art and implications for the imaging cardiologist. Neth Heart J. 2019;27(9):403-413.

26. Shah S. Precision Medicine for Heart Failure with Preserved Ejection Fraction: An Overview. J Cardiovasc Transl Res. 2017;10(3):233-244.

27. Lavrac N, Zupan B, Kononenko I, Kukar M, Keravnou E. Intelligent Data Analysis for Medical Diagnosis: Using Machine Learning and Temporal Abstraction.AI Commun.1998;11(3):191-218.

28. Hogarty D, Su J, Phan K, Attia M, Hossny M, Nahavandi S et al. Artificial Intelligence in Dermatology-Where We Are and the Way to the Future: A Review. Am J Clin Dermatol. 2019;21(1):41-47.

29. Hosny A, Parmar C, Quackenbush J, Schwartz L, Aerts H. Artificial intelligence in radiology.

30. Nat Rev Cancer. 2018;18(8):500-510.

31. Herzog H, Lerche C. Advances in Clinical PET/MRI Instrumentation. PET Clin. 2016;11(2):95-103.

32. Thrall J, Li X, Li Q, Cruz C, Do S, Dreyer K et al. Artificial Intelligence and Machine Learning in Radiology: Opportunities, Challenges, Pitfalls, and Criteria for Success. J Am Coll Radiol. 2018;15(3):504-508. 
33. Pagnozzi A, Fripp J, Rose S. Quantifying deep grey matter atrophy using automated segmentation approaches A systematic review of structural MRI studies. NeuroImage. 2019;201:116018.

34. Liu X, Zhou H, Hu Z, Jin Q, Wang J, Ye B. Clinical Application of Artificial Intelligence Recognition Technology in the Diagnosis of Stage T1 Lung Cancer. Canc. . 2019 ;22(5):319-323.

35. Schork N. Artificial Intelligence and Personalized Medicine. Cancer Treat Res. 2019;178:265-283.

36. Henstock P. Artificial Intelligence for Pharma: Time for Internal Investment. Trends Pharmacol Sci. 2019;40(8):543-546.

37. Zhong Y, Ru C, Zhang B, Cheng Y. Studies on the methodology for quality control in Chinese medicine manufacturing process based on the knowledge graph. Chi Mat Med. 2019 Dec;44(24) 5269-5276.

38. Duch W, Swaminathan K, Meller J. Artificial Intelligence Approaches for Rational Drug Design and Discovery. Curr Pharm Des. 2007;13(14):1497-1508.

39. Brasil S, Pascoal C, Francisco R, dos Reis Ferreira V, A. Videira P, Valadão G. Artificial Intelligence (AI) in Rare Diseases: Is the Future Brighter?. Genes(Basel). 2019;10(12):978.

40. Makino M, Yoshimoto R, Ono M, Itoko T, Katsuki T, Koseki A et al. Artificial intelligence predicts the progression of diabetic kidney disease using big data machine learning. Sci Rep. 2019;9(1).

41. Xiao C, Choi E, Sun J. Opportunities and challenges in developing deep learning models using electronic health records data: a systematic review. J Am Med Inform Assoc. 2018;25(10):14191428.

42. Shickel B, Tighe P, Bihorac A, Rashidi P. Deep EHR: A Survey of Recent Advances in Deep Learning Techniques for Electronic Health Record (EHR) Analysis. IEEE J Biomed Health Inform. 2018;22(5):1589-1604.

43. Corey K, Kashyap S, Lorenzi E, Lagoo-Deenadayalan S, Heller K, Whalen K et al. Development and validation of machine learning models to identify high-risk surgical patients using automatically curated electronic health record data (Pythia): A retrospective, single-site study. PLOS Med. 2018;15(11):e1002701.

44. Komorowski M, Celi L, Badawi O, Gordon A, Faisal A. The Artificial Intelligence Clinician learns optimal treatment strategies for sepsis in intensive care. Nat Med. 2018;24(11):1716-1720.

45. Benke K, Benke G. Artificial Intelligence and Big Data in Public Health. Int J Environ Res Public Health. 2018;15(12):2796.

46. Thiébaut R, Cossin S. Artificial Intelligence for Surveillance in Public Health. Yearb Med Inform 2019;28(01):232-234.

47. Kannan S, Shaik Syed Ali P, Sheeza A, Hemalatha K. COVID-19 (Novel Coronavirus 2019) - recent trends. Eur Rev Med Pharmacol Sci. 2020;24(4):2006-2011.

48. Li L, Qin L, Xu Z, Yin Y, Wang X, Kong B et al. Using Artificial Intelligence to Detect COVID-19 and Community-acquired Pneumonia Based on Pulmonary CT: Evaluation of the Diagnostic Accuracy. Radiology. 2020;296(2):E65-E71.
49. Ahuja A, Reddy V, Marques O. Artificial intelligence and COVID-19: A multidisciplinary approach. Integr Med Res. 2020;9(3):100434.

50. Salama M, Hassanien A, Mostafa A. The prediction of virus mutation using neural networks and rough set techniques. EURASIP J Bioinform Syst Biol. 2016;2016(1).

51. Jiang X, Coffee M, Bari A, Wang J, Jiang X, Huang J et al. Towards an Artificial Intelligence Framework for Data-Driven Prediction of Coronavirus Clinical Severity. Comp, Mat Cont. 2020;62(3):537-551.

52. Russo G, Reche P, Pennisi M, Pappalardo F. The combination of artificial intelligence and systems biology for intelligent vaccine design. Expert Opin Drug Discov. 2020;15(11):1267-1281.

53. Agbehadji I, Awuzie B, Ngowi A, Millham R. Review of Big Data Analytics, Artificial Intelligence and Nature-Inspired Computing Models towards Accurate Detection of COVID-19 Pandemic Cases and Contact Tracing. Int J Environ Res Public Health. 2020;17(15):5330.

54. Zadgaonkar A, Zadgaonkar A. System and method for estimating hormone level and physiological conditions by analysing speech samples. US20180322893, 2018.

55. Zadgaonkar A, Zadgaonkar A. System and method for estimating properties and physiological conditions of organs by analysing speech samples. 20180336918, 2018.

56. Li Y, Hannaford B, Rosen J. The Raven Open Surgical Robotic Platforms: A Review and Prospect. Acta Pol Hung. 2019;16(8): 35-51.

57. Carr E, Thomas V. Anticipating and experiencing postoperative pain: the patients' perspective. J Clin Nurs. 1997;6(3):191-201.

58. Raghavendra U, Acharya U, Adeli H. Artificial Intelligence Techniques for Automated Diagnosis of Neurological Disorders. Eur Neurol. 2019;82(1-3):41-64.

59. Marinovic N. The Wigner distribution and the ambiguity function: generalisations, enhancement, compression and some applications (signal processing, pattern recognition, computer vision, medical ultrasonics).1987: 1203-1203.

60. Shanahan J, Dai L. Introduction to Computer Vision and RealTime Deep Learning-based Object Detection. Proceedings of the 26th ACM SIGKDD Int Conf Knowl Discov Data Min. 2020;.

61. Hassan M, Malik A, Fofi D, Saad N, Karasfi B, Ali Y et al. Heart rate estimation using facial video: A review. Biomed Sig Proc Cont. 2017;38:346-360.

62. Pranata Y, Wang K, Wang J, Idram I, Lai J, Liu J et al. Deep learning and SURF for automated classification and detection of calcaneus fractures in CT images. Comp Met Prog Biomed. 2019;171:27-37.

63. Gerig G, Kuoni W, Kikinis R, Kübler O. Medical Imaging and Computer Vision: An integrated approach for diagnosis and planning. Mustererkennung 1989. 1989;219:425-432. 